\title{
ONE-DIMENSIONAL MODEL EQUATIONS FOR HYPERBOLIC FLUID FLOW
}

\author{
TAM DO, VU HOANG, MARIA RADOSZ, AND XIAOQIAN XU
}

\begin{abstract}
In this paper we study the singularity formation for two nonlocal 1D active scalar equations, focusing on the hyperbolic flow scenario. Those 1D equations can be regarded as simplified models of some $2 \mathrm{D}$ fluid equations.
\end{abstract}

\section{INTRODUCTION}

The following transport equation

$$
\omega_{t}+u \cdot \nabla \omega=0
$$

is a basic mathematical model in fluid dynamics. If $u$ depends on $\omega,(1)$ is called an active scalar equation. The problem of deciding whether blowup can occur for smooth initial data becomes very hard if the dependence of $\omega$ is nonlocal in space.

The relationship expressing $u$ in terms of $\omega$ is commonly called Biot-Savart law. We have the following examples in 2D:

$$
u=\nabla^{\perp}(-\triangle)^{-1} \omega
$$

where $\nabla^{\perp}=\left(-\partial_{y}, \partial_{x}\right)$ is the perpendicular gradient. Equations (1) and (2) are the vorticity form of $2 \mathrm{D}$ Euler equation. When we take

$$
u=\nabla^{\perp}(-\Delta)^{-\frac{1}{2}} \omega
$$

(1) becomes the surface quasi-geostrophic (SQG) equation, which has important applications in geophysics, or can be regarded as a toy model for the 3D-Euler equations. For more details we refer to [4].

A question of great importance is whether solutions for these equations form singularities in finite time. A promising new approach for the construction of singular solutions is to use the hyperbolic flow scenario. In [7], [8], such a scenario was proposed to obtain singular solutions for the 3D Euler equations, and in [10], the long-standing question of existence of solutions to the 2D Euler with double-exponential gradient growth was settled using hyperbolic flow.

The hyperbolic flow scenario in two dimensions can be explained in the following way. Consider e.g. a flow in the upper half-plane $\left\{x_{2}>0\right\}$. The essential properties required are (see Figure 1 for an illustration):

- There is a stagnant point of the flow at one boundary point (e.g. the origin) for all times.

- Along the boundary, the flow is essentially directed towards that point for all times.

Date: August 5, 2015. 
Such flows can be created by imposing symmetry and other conditions on the initial data. For incompressible flows the stagnant point is a hyperbolic point of the velocity field, hence the name.

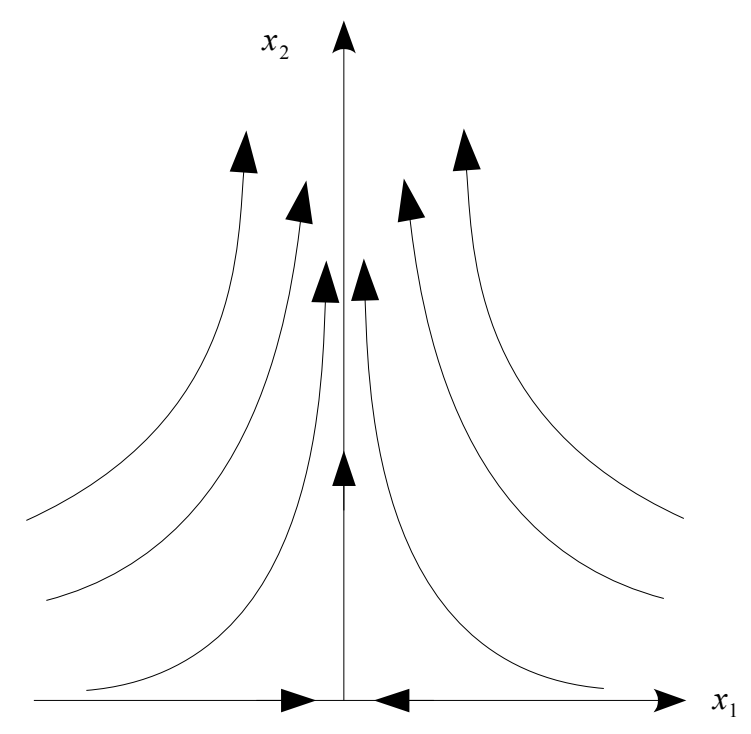

FigURE 1. Illustration of hyperbolic flow scenario in two dimensions.

The scenario is a natural candidate for creating flows with strong gradient growth or finite-time blowup, since the fluid is compressed along the boundary. Due to non-linear and non-local interactions however, the flow remains hard to control, so a rigourous proof of blowup for the 3D Euler equations using hyperbolic flow remains a challenge. The crucial issue is to stabilize the scenario up to the singular time.

One way to make progress in understanding and to gain insight into the hyperbolic blowup scenario is to study it in the context of one-dimensional model equations. This was begun in $[2,1]$, where one-dimensional models for the 2D-Boussinesq and 3D axisymmetric Euler equations were introduced and blowup was proven.

One-dimensional models capturing other aspects of fluid dynamical equations have a long-standing tradition, one of the earliest being the celebrated Constantin-Lax-Majda model [3]. We refer to the introduction of [11] for a more thorough review of known one-dimensional model equations, and to [1] for discussion of the aspects relating to the hyperbolic flow scenario.

In this paper, we will study $1 \mathrm{D}$ models of $(1)$ on $\mathbb{R}$ with the following two choices of $u$ :

$$
\begin{aligned}
u_{x} & =H \omega, \\
u & =(-\Delta)^{-\frac{\alpha}{2}} \omega=-c_{\alpha} \int_{\mathbb{R}}|y-x|^{-(1-\alpha)} \omega(y, t) d y .
\end{aligned}
$$

The choice (3) leads to a 1D analogue of the 2D Euler equation. This model is derived simply by restricting the dynamics to the boundary. In section 2 we give a brief heuristic argument which works by assuming that $\omega$ is concentrated in a small boundary layer. 
We note that the model (3) was mentioned in [1], where it was stated that (3) has properties analogous to the 2D Euler equation, without giving details. In particular, in [1] a 1D model of the 2D Boussinesq equations (an extended version of (3)) was introduced and studied. One of our goals here is to validate the 1D model introduced in [1] in a setting where comparison with $2 \mathrm{D}$ results are available. The fact shown below, that the solutions to the model problem (3) behave similarly to the full 2D Euler case, provides support to the usefulness of the extended version of this model in [1] for getting insight into behavior of solutions to 2D Boussinesq system and 3D Euler equation.

The model defined by (4) is called $\alpha$-patch model and appears in [6], where also a viscosity term is present. From the regularity standpoint, the $\alpha$-patch model is between $1 \mathrm{D}$ Euler $u_{x}=H \omega$ and the Córdoba-Córdoba-Fontelos model $u=H \omega$ (see $[5,11]$ ), which is an analogue of the SQG equation. These two models differ however from a geometric perspective, since the symmetry properties of the Biot-Savart laws are different. For the CCF model, the velocity field is odd for even $\omega$, whereas (4) is odd for odd $\omega$. It is important to choose data with the right symmetry to make $u$ odd, and thus to create a stagnant point of the flow at the origin for all times.

We note that local existence and blowup results for (4) were given in [6], where also dissipation is allowed. There the authors rely on a suitable Lyapunov function to show blowup, whereas we emphasize the more geometric aspects in this paper. That is, we will be studying the analogue of the hyperbolic flow scenario for the above 1D models and show that this leads to natural and intuitive constructions of solutions with strong gradient growth and finite-time blowup.

Another blowup result related to hyperbolic flow was recently proven by A. Kiselev, L. Ryzhik, Y. Yao and A. Zlatoš [9] and concerns a $\alpha$-patch model in 2D for small $\alpha>0$.

\section{EULER 1D MODEL}

2.1. Heuristic derivation. Recall the 2D Euler equations in vorticity form

$$
\omega_{t}+u \cdot \nabla \omega=0
$$

where $u=\nabla^{\perp}(-\Delta)^{-1} \omega$.

We first indicate a simple heuristic motivation for the choice (3) (see also [1]). Consider the 2D Euler equation in a half-space $\left\{x_{2} \geq 0\right\}$ and denote $\bar{x}=\left(x_{1},-x_{2}\right)$. The $x_{1^{-}}$ component of the velocity (up to a normalization constant) for compactly supported vorticity $\omega$ is given by

$$
u_{1}(x, t)=-\int_{\mathbb{R}^{2}} \frac{\left(y_{2}-x_{2}\right)}{|y-x|^{2}} \omega(y, t) d y
$$

where $\omega$ has been extended to $\left\{x_{2} \leq 0\right\}$ by odd reflection $(\omega(\bar{x}, t)=-\omega(x, t))$.

Suppose now that $\omega$ is concentrated in a boundary layer of width $a>0$ and that $\omega\left(x_{1}, x_{2}, t\right)=\omega\left(x_{1}, t\right)$ in this boundary layer. Then a calculation gives

$$
u_{1}\left(x_{1}, 0, t\right)=-2 \int_{\mathbb{R}} \log \left(\frac{\left(y_{1}-x_{2}\right)^{2}+a^{2}}{\left(y_{1}-x_{1}\right)^{2}}\right) \omega\left(y_{1}, t\right) d y_{1} .
$$


If we now retain only the singular part of the kernel $\log \left(\frac{z^{2}+a^{2}}{z^{2}}\right) \sim-2 \log |z|$ and identify $u$ with $u_{1}$, we get (dropping the constants)

$$
u(x, t)=\int_{\mathbb{R}} \log |y-x| \omega(y, t) d y .
$$

So a reasonable $1 \mathrm{D}$ model is

$$
\omega_{t}+u \omega_{x}=0, \quad u_{x}=H \omega, \quad \omega(x, 0)=\omega_{0}(x) \quad(x, t) \in \mathbb{R} \times[0, \infty) .
$$

where $H$ is the Hilbert transform, using the convention

$$
H \omega(x, t)=P . V . \int \frac{\omega(y, t)}{x-y} d y .
$$

For this model, we have the following local well-posedness property:

Proposition 2.1. Given initial data $\omega_{0} \in H_{0}^{m}((0,1))$ with $m \geq 2$, there exists $T=$ $T\left(\left\|\omega_{0}\right\|_{H_{0}^{m}}\right)>0$ such that the system has a unique classical solution $\omega \in C\left([0, T] ; H_{0}^{m}\right)$.

The proof is standard so we skip it here.

An alternative argument to motivate (3) is to observe that the gradient of the 2D Euler velocity is given by a zero-th order operator acting on $\omega$. In one dimension, this leaves only the choice $u_{x}=c H \omega$ or $u_{x}=c \omega, c$ being a nonzero constant. So we could also consider the model

$$
\omega_{t}+u \omega_{x}=0, \quad u_{x}=-\omega, \quad \omega(x, 0)=\omega_{0}(x) \quad(x, t) \in \mathbb{R} \times[0, \infty) .
$$

(8) is however not a close analogue of 2D Euler (see Remark 2.5).

2.2. Sharp a-priori bounds for gradient growth. We will first prove the global regularity of the solution to equation $(7)$ by showing that $\omega_{x}$ can grow at most with double exponential rate in time. Then we will give an example of a smooth solution to (7) where such growth of the gradient of $\omega$ is achieved, meaning the bound is sharp.

Due to the Biot-Savart law relating $u$ and $\omega$, the proof of an upper bound for $\left\|\omega_{x}(\cdot, t)\right\|_{\infty}$ is very similar to the proof for the full 2D Euler equations. For the reader's convenience, we give the proof. Recall first the definition of the Hölder norm

$$
\|\omega\|_{C^{\alpha}}=\sup _{|x-y| \leq 1, x \neq y} \frac{|\omega(x)-\omega(y)|}{|x-y|^{\alpha}}
$$

for compactly supported $\omega$.

We will need an estimate on the Hilbert transform:

Lemma 2.2. Let $0<\alpha<1$. Suppose supp $(\omega) \subset[-D(t), D(t)]$ and assume without loss of generality that $\left\|\omega_{0}\right\|_{L^{\infty}}=1$. Then

$$
\left\|u_{x}\right\|_{\infty} \leq C(\alpha)\left(1+|\log (D(t))|+\log \left(1+\|\omega\|_{C^{\alpha}}\right)\right)
$$

Proof. For any $\delta>0$, we have

$$
\left|\int_{[-D(t), D(t)] \backslash(x-\delta, x+\delta)} \frac{\omega(y)}{x-y} d y\right| \leq C \int_{\delta}^{D(t)} \frac{1}{y} d y \leq C(|\log \delta|+|\log (D(t))|) .
$$


Using the oddness of $\frac{1}{x}$, we have

$$
\left|P . V . \int_{x-\delta}^{x+\delta} \frac{\omega(y)}{x-y} d y\right|=\left|\int_{x-\delta}^{x+\delta} \frac{1}{x-y}(\omega(y)-\omega(x)) d y\right| \leq C(\alpha)\|\omega(x, t)\|_{C^{\alpha}} \delta^{\alpha} .
$$

Choosing $\delta=\min \left\{1,\left(\frac{1}{\|\omega\|_{C^{\alpha}}}\right)^{\frac{1}{\alpha}}\right\}$, we get the desired estimate of $\left\|u_{x}\right\|_{\infty}$.

The following Lemma gives an estimate on $D(t)$.

Lemma 2.3. Suppose the support of $\omega_{0}$ is in $[-1,1]$ and $\left\|\omega_{0}\right\|_{L^{\infty}}=1$. Then the support of $\omega(x, t)$ will be inside $\left[-C \exp \left(C e^{C t}\right), C \exp \left(C e^{C t}\right)\right]$, for some universal constant $C>0$.

Proof. Suppose supp $\omega=[-D(t), D(t)]$. Then for any point $x$ inside of this interval, we have

$$
|u(x)| \leq \int_{-D(t)}^{D(t)}|\log | x-y|| d y \leq C \int_{0}^{2 D(t)}|\log | s|| d s \leq C D(t)(|\log (D(t))|+1) .
$$

By following the trajectory of the particle at $D(t)$,

$$
D^{\prime}(t) \leq C D(t)(|\log (D(t))|+1) .
$$

A simple argument using differential inequalities shows that $D(t)$ is always less than $z(t)$, where $z(t)$ is the solution of

$$
z^{\prime}(t)=C z(t)(\log z(t)+1), z(0)=\min \{D(0), 2\} .
$$

This yields the double-exponential upper bound on $D(t)$.

The following Theorem gives the double exponential upper bound for $\omega_{x}$.

Theorem 2.4. There is universal constant $C$ such that if $\omega_{0}$ is smooth, compactly supported with supp $\omega_{0} \subset[-1,1]$ and $\|\omega\|_{L^{\infty}}=1$,

$$
\log \left(1+\left\|\omega_{x}\right\|_{L^{\infty}}\right) \leq C \log \left(1+\left\|\left(\omega_{0}\right)_{x}\right\|_{L^{\infty}}\right) e^{C t} \quad(t \geq 0) .
$$

Proof. We follow the proof in [10]. Let us denote the flow map corresponding to the evolution by $\Phi_{t}(x)$. Then

and

$$
\frac{\partial}{\partial t} \Phi_{t}(x)=u\left(\Phi_{t}(x), t\right), \quad \Phi_{0}(x)=x
$$

$$
\left|\frac{\partial_{t}\left|\Phi_{t}(x)-\Phi_{t}(y)\right|}{\left|\Phi_{t}(x)-\Phi_{t}(y)\right|}\right| \leq\left\|u_{x}\right\|_{L^{\infty}} .
$$

After integration, and by Lemma 2.2 and Lemma 2.3, this gives

$$
f(t)^{-1} \leq \frac{\left|\Phi_{t}(x)-\Phi_{t}(y)\right|}{|x-y|} \leq f(t)
$$

where

$$
f(t)=\exp \left(C \int_{0}^{t}\left(1+\exp (C s)+\log \left(1+\left\|\omega_{x}\right\|_{L^{\infty}}\right)\right) d s\right) .
$$

This bound also holds for $\Phi_{t}^{-1}$. On the other hand,

$$
\left\|\omega_{x}\right\|_{L^{\infty}}=\sup _{x \neq y} \frac{\left|\omega_{0}\left(\Phi_{t}^{-1}(x)\right)-\omega_{0}\left(\Phi_{t}^{-1}(y)\right)\right|}{|x-y|} \leq\left\|\left(\omega_{0}\right)_{x}\right\| \sup _{x \neq y} \frac{\left|\Phi_{t}^{-1}(x)-\Phi_{t}^{-1}(y)\right|}{|x-y|} .
$$


Which means we have

$$
\left(1+\left\|\omega_{x}\right\|_{L^{\infty}}\right) \leq\left(1+\left\|\left(\omega_{0}\right)_{x}\right\|_{L^{\infty}}\right) \exp \left(C \int_{0}^{t} 1+\exp (C s)+\log \left(1+\left\|\omega_{x}\right\|_{L^{\infty}}\right) d s\right),
$$

or

$$
\log \left(1+\left\|\omega_{x}\right\|_{L^{\infty}}\right) \leq \log \left(1+\left\|\left(\omega_{0}\right)_{x}\right\|_{L^{\infty}}\right)+C \exp (C t)+C \int_{0}^{t}\left(1+\log \left(1+\left\|\omega_{x}\right\|_{L^{\infty}}\right)\right) d s .
$$

So $y(t):=\log \left(1+\left\|\omega_{x}\right\|_{L^{\infty}}\right)$ satisfies the integral inequality

$$
y^{\prime}(t) \leq y(0)+C e^{C t}+\int_{0}^{t}(1+y(s)) d s
$$

and by the integral form Gronwall's inequality and some elementary manipulations, we arrive at the bound $y(t) \leq C_{1} y(0) e^{C_{2} t}$. This yields the desired bound on $\left\|\omega_{x}\right\|_{\infty}$.

Remark 2.5. If we choose our Biot-Savart law to be $u_{x}=-\omega$, then from a modification of the above proof we get an exponential upper bound for $\left\|\omega_{x}\right\|_{L^{\infty}}$. This is different from the 2D Euler equation, which suggests that (7) is a better analogue of the 2D Euler equation than (8). Moreover the equation (8) also has different symmetry properties.

Next we construct initial data $\omega_{0}$ such that $\left\|\omega_{x}(\cdot, t)\right\|_{L^{\infty}}$ grows with double-exponential rate, proving the sharpness of the a-priori bound (9). The hyperbolic flow scenario is created in the following way: First, we require that the initial data $\omega_{0}$ is odd with respect to the origin, and has compact support. By Proposition 2.1, the oddness is easily seen to be preserved by the evolution. Consequently, the velocity field (which is also an odd function) can be written as

$$
u(x, t)=-x \int_{0}^{\infty} K\left(\frac{x}{y}\right) \frac{\omega(y, t)}{y} d y \quad(x>0)
$$

where

$$
K(s):=\frac{1}{s} \log \left|\frac{s+1}{s-1}\right| .
$$

Note that the origin is a stagnant point of the flow for all times. By taking $\omega_{0}$ to be positive on the right, the direction of the flow is towards the origin. More precisely, $\omega_{0}$ is defined as follows (see Figure 2):

- Let $\omega_{0}$ be supported on $[-1,1]$, smooth and odd. Choose numbers $0<x_{1}(0)<$ $2 x_{2}(0)<1$ such that $M x_{1}(0) \leq x_{2}(0)$ where $M$ will be determined later. Require that $\omega_{0}$ is increasing on $\left[0, x_{1}(0)\right]$, decreasing on $\left[x_{2}(0), 1\right]$ and identically 1 on $\left[x_{1}(0), x_{2}(0)\right]$.

Using the earlier notation $\Phi_{t}$ for the flow map associated to $(7)$, let

$$
\begin{aligned}
& x_{1}(t):=\Phi_{t}\left(x_{1}(0)\right) \\
& x_{2}(t):=\Phi_{t}\left(x_{2}(0)\right)
\end{aligned}
$$

It is easy to see that the general structure of $\omega_{0}$ will be preserved by the flow: For fixed $t, \omega(x, t)$ will be increasing on $\left[0, x_{1}(t)\right]$, decreasing on $\left[x_{2}(t), 1\right]$ and identically 1 on $\left[x_{1}(t), x_{2}(t)\right]$. In fact, since $u(x, t) \leq 0$ for $x \geq 0, x_{1}(t)$ and $x_{2}(t)$ will be moving towards 


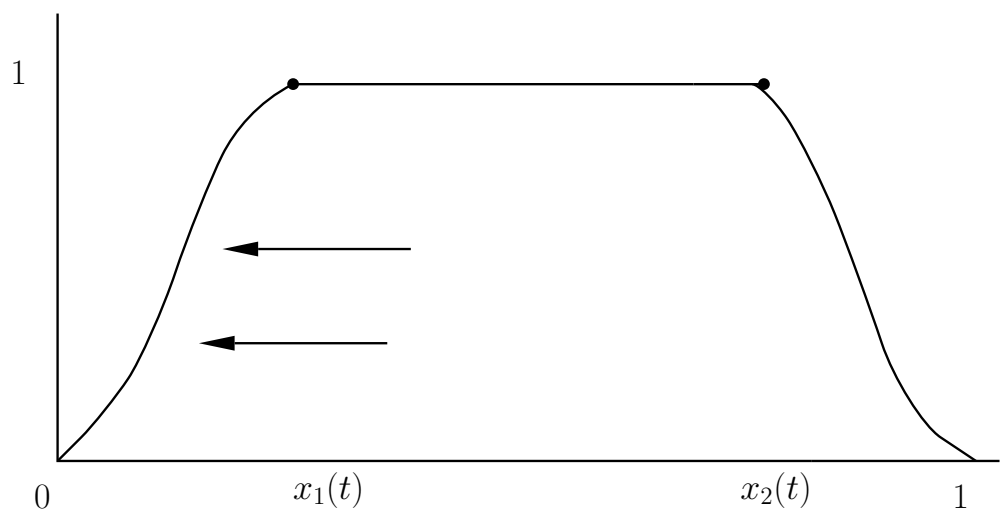

Figure 2. Structure of $\omega(x, t)$.

the origin in time. We will show that the quantity $\frac{x_{2}(t)}{x_{1}(t)}$ increases double exponentially in time. This is sufficient to conclude the desired growth of $\left\|\omega_{x}(\cdot, t)\right\|_{L^{\infty}}$.

Theorem 2.6. Assume our initial data is defined as above, then

$$
\log \frac{x_{2}(t)}{x_{1}(t)} \geq \log \frac{x_{2}(0)}{x_{1}(0)} \exp (C t) \quad(t>0)
$$

for some positive constant $C$. As a consequence,

$$
\log \left\|\omega_{x}(\cdot, t)\right\|_{L^{\infty}} \geq C_{1} \exp \left(C_{2} t\right) \quad(t>0)
$$

for some $C_{1}, C_{2}>0$.

Theorem 2.4 quickly follows from the following Lemma:

Lemma 2.7. Suppose $1 \geq x_{2} \geq 8 x_{1}$. There are universal constants $C_{0}$ and $C_{1}$ so that

$$
\frac{d}{d t}\left(\frac{x_{2}}{x_{1}}\right) \geq C_{1} \frac{x_{2}}{x_{1}}\left(\log \left(\frac{x_{2}}{x_{1}}\right)-C_{0}\right)
$$

Proof. First observe

$$
\begin{aligned}
\frac{d}{d t}\left(\frac{x_{2}}{x_{1}}\right) & =\frac{x_{2}^{\prime} x_{1}-x_{1}^{\prime} x_{2}}{x_{1}^{2}}=\frac{u\left(x_{2}\right) x_{1}-u\left(x_{1}\right) x_{2}}{x_{1}^{2}}=\frac{x_{2}}{x_{1}}\left(\frac{u\left(x_{2}\right)}{x_{2}}-\frac{u\left(x_{1}\right)}{x_{1}}\right) \\
& =\frac{x_{2}}{x_{1}} \int_{0}^{1}\left[K\left(\frac{x_{1}}{y}\right)-K\left(\frac{x_{2}}{y}\right)\right] \frac{\omega(y)}{y} d y .
\end{aligned}
$$


We decompose the integral into 4 pieces which we will estimate separately:

$$
\begin{aligned}
& \int_{0}^{1}\left[K\left(\frac{x_{1}}{y}\right)-K\left(\frac{x_{2}}{y}\right)\right] \frac{\omega(y)}{y} d y \\
= & \int_{0}^{2 x_{1}}+\int_{2 x_{1}}^{\frac{1}{2} x_{2}}+\int_{\frac{1}{2} x_{2}}^{2 x_{2}}+\int_{2 x_{2}}^{1}\left[K\left(\frac{x_{1}}{y}\right)-K\left(\frac{x_{2}}{y}\right)\right] \frac{\omega(y)}{y} d y \\
= & I+I I+I I I+I V .
\end{aligned}
$$

For $I$, we use $0 \leq \omega(y) \leq 1$ and $2 x_{1} \leq x_{2} \leq 1$ :

$$
\begin{aligned}
0 \leq I & \leq \int_{0}^{2 x_{1}} \frac{1}{x_{1}} \log \frac{\left(x_{1}+y\right)}{\left|x_{1}-y\right|} d y+\int_{0}^{2 x_{1}} \frac{1}{x_{2}} \log \frac{\left(x_{2}+y\right)}{\left|x_{2}-y\right|} d y \\
& =\frac{1}{x_{1}} 3 x_{1} \log 3+\frac{1}{x_{2}}\left[2 x_{1} \log \frac{1+\frac{2 x_{1}}{x_{2}}}{1-\frac{2 x_{1}}{x_{2}}}+x_{2} \log \left(1-\frac{2 x_{1}}{x_{2}}\right)+x_{2} \log \left(1+\frac{2 x_{1}}{x_{2}}\right)\right] \\
& \leq 3 \log 3+2 \log 2 .
\end{aligned}
$$

Using the fact that $K(s)$ is increasing in $[0,1)$ and decreasing in $(1, \infty]$ and that $\omega(y)=1$ for $y \in\left(2 x_{1}, \frac{1}{2} x_{2}\right)$ we get

$$
\begin{aligned}
I I & =\int_{2 x_{1}}^{\frac{1}{2} x_{2}}\left[K\left(\frac{x_{1}}{y}\right)-K\left(\frac{x_{2}}{y}\right)\right] \frac{\omega(y)}{y} d y \geq \int_{2 x_{1}}^{\frac{1}{2} x_{2}}\left(2-\frac{1}{2} \log (3)\right) \frac{1}{y} d y \\
& =\left(2-\frac{1}{2} \log (3)\right) \log \left(\frac{x_{2}}{x_{1}}\right)-C .
\end{aligned}
$$

Using the positivity of $K$,

$$
\begin{aligned}
I I I & =\int_{\frac{1}{2} x_{2}}^{2 x_{2}}\left[K\left(\frac{x_{1}}{y}\right)-K\left(\frac{x_{2}}{y}\right)\right] \frac{\omega(y)}{y} d y \geq-\int_{\frac{1}{2} x_{2}}^{2 x_{2}} K\left(\frac{x_{2}}{y}\right) \omega(y) \frac{1}{y} d y \\
& \geq-\int_{\frac{1}{2}}^{2} \frac{1}{s^{2}} \log \frac{|s+1|}{|s-1|} d s \geq-C .
\end{aligned}
$$

We estimate $I V$ in the following way, using that $\omega(y) \leq 1$ and $\frac{x_{1}}{y} \leq \frac{x_{2}}{y} \leq 1$ for $2 x_{2} \leq y \leq 1$ :

$$
\begin{aligned}
|I V| & =\left|\int_{2 x_{2}}^{1}\left[K\left(\frac{x_{1}}{y}\right)-K\left(\frac{x_{2}}{y}\right)\right] \frac{\omega(y)}{y} d y\right| \leq \int_{2 x_{2}}^{1}\left[K\left(\frac{x_{2}}{y}\right)-K\left(\frac{x_{1}}{y}\right)\right] \frac{1}{y} d y \\
& \leq \int_{2 x_{2}}^{1} \frac{1}{x_{2}} \log \frac{y+x_{2}}{y-x_{2}} d y-\int_{2 x_{2}}^{1} \frac{1}{x_{1}} \log \frac{y+x_{1}}{y-x_{1}} \\
& =(i)-(i i) .
\end{aligned}
$$

We can compute $(i)$ directly and get

$$
(i)=\frac{1}{x_{2}} \log \frac{1+x_{2}}{1-x_{2}}+\log \left(1+x_{2}\right)\left(1-x_{2}\right)-2 \log \left(x_{2}\right)-3 \log (3) .
$$

Similarly, for $(i i)$, we have

$$
(\text { ii })=\frac{1}{x_{1}} \log \frac{1+x_{1}}{1-x_{1}}+\log \left(x_{1}+1\right)\left(1-x_{1}\right)-2 \frac{x_{2}}{x_{1}} \log \frac{2 x_{2}+x_{1}}{2 x_{2}-x_{1}}-\log \left(2 x_{2}+x_{1}\right)\left(2 x_{2}-x_{1}\right) .
$$


Note that in the expressions for $(i)$ and $(i i)$, all terms can be bounded by universal constants except for $-2 \log \left(x_{2}\right)$ and $\log \left(2 x_{2}+x_{1}\right)\left(2 x_{2}-x_{1}\right)$. However, using $x_{1}<x_{2}$, we get

$$
|I V| \leq C-2 \log \left(x_{2}\right)+\log \left(2 x_{2}+x_{1}\right)\left(2 x_{2}-x_{1}\right)=C+\log \left(4-\left(\frac{x_{1}}{x_{2}}\right)^{2}\right) \leq C .
$$

The proof of Theorem 2.6 is now completed as follows: choose $M>8$ so large such that $\frac{1}{2} \log (M)-C_{0} \geq 0$. We have thus $\frac{1}{2} \log \left(\frac{x_{2}^{0}}{x_{1}^{0}}\right)-C_{0} \geq 0$. From Lemma 2.7 it follows that $\frac{x_{2}(t)}{x_{1}(t)}$ is growing in time and that we have

$$
\frac{d}{d t}\left(\frac{x_{2}}{x_{1}}\right) \geq \frac{C_{1}}{2} \frac{x_{2}}{x_{1}} \log \left(\frac{x_{2}}{x_{1}}\right)
$$

or $\frac{d}{d t} \log \left(\frac{x_{2}}{x_{1}}\right) \geq \frac{C_{1}}{2} \log \left(\frac{x_{2}}{x_{1}}\right)$ for all times. This clearly implies that $\frac{x_{2}}{x_{1}}$ grows doubleexponentially.

Remark 2.8. In [10], the Biot-Savart law is decomposed into a main contribution and an error term. In our case (10), the main contribution would be

$$
-x \int_{x}^{\infty} \frac{\omega(y)}{y} d y
$$

If we replace (10) by (12), then double-exponential growth of $\frac{x_{2}}{x_{1}}$ can be proven by a straightforward argument. In this case, the computation for the estimate in Lemma 2.7 becomes much easier.

\section{3. $\alpha$-PATCH $1 \mathrm{D}$ MODEL}

In this section, we consider the 1D model equation

$$
\omega_{t}+u \omega_{x}=0
$$

with a different Biot-Savart law

$$
u(x, t)=(-\Delta)^{-\alpha / 2} \omega(x, t)=-c_{\alpha} \int_{\mathbb{R}}|y-x|^{-(1-\alpha)} \omega(y, t) d y, \quad \alpha \in(0,1)
$$

For convenience, we will assume the constant $c_{\alpha}$ associated with the fractional Laplacian is 1 , and we write $\gamma=1-\alpha$.

This problem has been studied in [6], where local existence and uniqueness results for smooth initial data are proven. From these, we can show that this equation preserves oddness and $u(0, t)=0$ holds with odd initial datum. For odd data, we can write

$$
u(x, t)=-\int_{0}^{\infty} k(x, y) \omega(y, t) d y
$$

where $k(x, y)=|y-x|^{-\gamma}-|y+x|^{-\gamma}$. Note that $k(x, y) \geq 0$ for $x \neq y \in(0, \infty)$.

Following similar ideas as for 1D Euler, we specifiy our initial data $\omega_{0}$ as follows: 
- Pick $0<x_{1}(0), x_{2}(0)$ with $M x_{1}(0)<x_{2}(0)$. Let $\omega_{0}$ be smooth, odd, $\omega_{0}(x) \geq 0$ for $x>0$ and have its support in $\left[-2 x_{2}(0), 2 x_{2}(0)\right] . M>1$ is to be chosen below. Let $\omega_{0}$ moreover be bounded by 1 , smoothly increasing in the interval $\left[0, x_{1}(0)\right]$ and $\omega_{0}=1$ between $x_{1}(0)$ and $x_{2}(0)$.

As long as the solution remains smooth, the general structure of the solution does not change. Let $x_{1}(t), x_{2}(t)$ be again the position of the particles starting at $x_{1}(0), x_{2}(0)$.

Theorem 3.1. There exists a choice of $x_{1}(0), x_{2}(0), M$ and a time $T>0$ such that the smooth solution of (13) for the above initial data cannot be continued beyond T. Provided the solution remains smooth on the time interval $[0, T)$, the particle starting at $x_{1}(0)$ reaches the origin at time $t=T$, i.e.

$$
\lim _{t \rightarrow T} x_{1}(t)=0 .
$$

In this sense, the solution forms a "shock".

Remark 3.2. In [6], the existence of blowup solutions to (13) is shown using energy methods. The advantage is that they are able to include a dissipation term. However, it is difficult to see the geometric blowup mechanism clearly using energy methods. Our proof for the inviscid case uses the dynamics of the solution and gives a more intuitive picture of the blowup, and is easily generalized to other even kernels having the same singular behavior.

In the rest of this section, we will prove Theorem 3.1. So assume that for arbitrary choice of $x_{1}(0), x_{2}(0), M$, we have a smooth solution $\omega$ defined for all times $t \in[0, \infty)$.

First of all, we track the movement of the particle starting at $x_{1}(0)$, which is the following Lemma.

Lemma 3.3. There exists a universal constant $M>2$ so that if $M x_{1}(t) \leq x_{2}(t)$, the velocity at $x_{1}(t)$ will satisfy

$$
u\left(x_{1}(t), t\right) \leq-C x_{1}(t)^{1-\gamma},
$$

for some universal constant $C$.

Proof. Let $u_{1}=u\left(x_{1}(t), t\right)$. Since $k, \omega \geq 0$ on $(0, \infty)$

$$
\begin{aligned}
-u_{1} & \geq \int_{2 x_{1}}^{x_{2}} k\left(x_{1}, y\right) d y \\
& =c_{\gamma}\left[-\left(x_{2}+x_{1}\right)^{1-\gamma}+\left(x_{2}-x_{1}\right)^{1-\gamma}+\left(3 x_{1}\right)^{1-\gamma}-x_{1}^{1-\gamma}\right] \\
& =c_{\gamma}\left[\left(3^{1-\gamma}-1\right) x_{1}^{1-\gamma}+\left(x_{2}-x_{1}\right)^{1-\gamma}-\left(x_{2}+x_{1}\right)^{1-\gamma}\right] \\
& =c_{\gamma} x_{1}^{1-\gamma}\left[\left(3^{1-\gamma}-1\right)+\frac{1}{x_{1}^{1-\gamma}}\left(\left(x_{2}-x_{1}\right)^{1-\gamma}-\left(x_{2}+x_{1}\right)^{1-\gamma}\right)\right]
\end{aligned}
$$

for some constant $c_{\gamma}>0$. Note that $\left(3^{1-\gamma}-1\right)>0$. We can write

$$
\begin{aligned}
\frac{1}{x_{1}^{1-\gamma}}\left(x_{2}-x_{1}\right)^{1-\gamma}-\left(x_{2}+x_{1}\right)^{1-\gamma} & =\frac{x_{2}^{1-\gamma}}{x_{1}^{1-\gamma}}\left[\left(1-\frac{x_{1}}{x_{2}}\right)^{1-\gamma}-\left(1+\frac{x_{1}}{x_{2}}\right)^{1-\gamma}\right] \\
& =: \frac{x_{2}^{1-\gamma}}{x_{1}^{1-\gamma}} f\left(x_{1} / x_{2}\right) .
\end{aligned}
$$


There exists a constant $C>0$ with $\left|f\left(x_{1} / x_{2}\right)\right| \leq C\left|x_{1} / x_{2}\right|$ for $\left|x_{1} / x_{2}\right| \leq 1 / 2$, and so

$$
-u_{1} \geq c_{\gamma} x_{1}^{1-\gamma}\left[\left(3^{1-\gamma}-1\right)-C M^{-\gamma}\right]
$$

if $M x_{1}(t) \leq x_{2}(t)$. Now choose $M$ large enough so that $C M^{-\gamma}$ is smaller than the number $\frac{1}{2}\left(3^{1-\gamma}-1\right)$.

This estimate of velocity field will lead to a blowup in finite time, provided we can show $M x_{1}(t) \leq x_{2}(t)$. More precisely,

$$
\frac{d}{d t} x_{1}(t)=u\left(x_{1}\right) \leq-C x_{1}^{1-\gamma}
$$

implying

$$
x_{1} \leq C\left(x_{1}(0)^{\gamma}-C t\right)^{\frac{1}{\gamma}} .
$$

This shows that no later than $T_{0}:=C^{-1} x_{1}(0)^{\gamma}$, the particle $x_{1}(t)$ will reach the origin, and the solution cannot be continued smoothly. Note that $T_{0}$ does not depend on $x_{2}(0)$.

It remains therefore to control the motion of $x_{2}(t)$, concluding the proof.

Lemma 3.4. For $x_{2}(0)$ large enough, $M x_{1}(t)<x_{2}(t)$ for $t \in\left[0, T_{0}\right)$.

Proof. We write $u\left(x_{2}(t), t\right)=u_{2}$. Observe that the support of $\omega(\cdot, t)$ is always contained in $\left[-2 x_{2}(0), 2 x_{2}(0)\right]$ because of $u(x, t) \leq 0$ for $x>0$.

Next we find an upper bound on $u_{2}$ :

$$
\left|u_{2}(t)\right| \leq \int_{-2 x_{2}(0)}^{2 x_{2}(0)}|y-x|^{-\gamma} \leq C x_{2}(0)^{1-\gamma} .
$$

Hence,

$$
x_{2}(t) \geq x_{2}(0)-\int_{0}^{T_{0}}\left|u_{2}(s)\right| d s \geq x_{2}(0)\left(1-C x_{2}(0)^{-\gamma} T_{0}\right) .
$$

Now choose $x_{2}(0)$ so large that $M x_{1}(0)<x_{2}(0)\left(1-C x_{2}(0)^{-\gamma} T_{0}\right)$. But then

$$
M x_{1}(t) \leq M x_{1}(0)<x_{2}(0)\left(1-C x_{2}(0)^{-\gamma} T_{0}\right) \leq x_{2}(t),
$$

giving the statement of the Lemma.

Acknowledgments. The authors would like to thank Prof. Alexander Kiselev for helpful discussions. TD would like to acknowledge the support of NSF grant 1453199 . VH expresses his gratitude to the German Research Foundation (DFG) for continued support through grants FOR 5156/1-1 and FOR 5156/1-2. VH also acknowledges partial support by NSF grant NSF-DMS 1412023. XX would like to acknowledge the support of NSF grant 1535653 .

\section{REFERENCES}

[1] Kyudong Choi, Thomas Y. Hou, Alexander Kiselev, Guo Luo, Vladimir Šverák, and Yao Yao. On the finite-time blowup of a 1d model for the 3d axisymmetric Euler equations. arXiv:1407.4776, 2014.

[2] Kyudong Choi, Alexander Kiselev, and Yao Yao. Finite time blow up for a 1d model of 2d Boussinesq system. Comm. Math. Phys., 334(3):1667-1679, 2015.

[3] P. Constantin, P.D. Lax, and A. Majda. A simple one-dimensional model for the three-dimensional vorticity equation. Comm. Pure Appl. Math., 38:715-724, 1985. 
[4] Peter Constantin, Andrew J Majda, and Esteban Tabak. Formation of strong fronts in the 2-d quasigeostrophic thermal active scalar. Nonlinearity, 7(6):1495-1533, 1994.

[5] A. Córdoba, D. Córdoba, and M.A. Fontelos. Formation of singularities for a transport equation with nonlocal velocity. Ann. of Math.(2), 162(3):1377-1389, 2005.

[6] Hongjie Dong and Dong Li. On a one-dimensional $\alpha$-patch model with nonlocal drift and fractional dissipation. Trans. Amer. Math. Soc., 366(4):2041-2061, 2014.

[7] Thomas Y. Hou and Guo Luo. Toward the finite-time blowup of the 3d axisymmetric Euler equations: A numerical investigation. Multiscale Model. Simul., 12(4):1722-1776, 2014.

[8] Thomas Y. Hou and Guo Luo. Potentially singular solutions of the 3D axisymmetric Euler equations. PNAS, vol. 111 no. 36, 12968-12973, DOI 10.1073/pnas.1405238111.

[9] Alexander Kiselev, Lenya Ryzhik, Yao Yao, and Andrej Zlatoš. Finite-time blow up in $\alpha$-patch model. In preparation, 2015.

[10] Alexander Kiselev and Vladimir Šverák. Small scale creation for solutions of the incompressible two dimensional Euler equation. Ann. of Math.(2), 180(3):1205-1220, 2014.

[11] Luis Silvestre and Vlad Vicol. On a transport equation with nonlocal drift. arXiv:1408.1056, 2014.

Tam Do, Rice University, Department of Mathematics-MS 136, Box 1892, Houston, TX 77251-1892

E-mail address: tam.do@rice.edu

Vu Hoang, Rice University, Department of Mathematics-MS 136, Box 1892, Houston, TX 77251-1892

E-mail address: Vu.Hoang@rice.edu

Maria Radosz, Rice University, Department of Mathematics-MS 136, Box 1892, HousTON, TX 77251-1892

E-mail address: maria_radosz@hotmail.com

Xiaoqian Xu, Department of Mathematics, University of Wisconsin, Madison, Wi 53706

E-mail address: xxu@math.wisc.edu 